\title{
Novel Space-Time Processing of DS/CDMA Multipath Signal
}

\author{
Kwang-Cheng Chen, Wei-Chiang Wu \\ Institute of Communications Engineering, College of Electrical Engineering, National Taiwan University, Taipei, R.O.C.
}

\begin{abstract}
This paper proposes a novel space-time receiver structure that jointly combines all possible diversities to combat multipathinduced interchip interference (ICI) and multiucers-induced cochannel interference (CCI or MAI). The proposed structure converts the design of a 2D RAKE receiver into the deconvolution filtering or space-time beamforming framework. The linear complexity detection algorithm is both computationally and conceptually feasible. The effect of pointing error (estimation error of the channel parameters) on system performance is also extensively studied. Moreover, we propose an adaptive robust beamforming technique to improve system performance that is severely degraded in the presence of pointing error. Simulation results shows that the proposed algorithm can effectively improve system performance to approach the ideal case (without pointing error).
\end{abstract}

\section{Introduction}

The RAKE receiver first proposed by Price and Green [1] is a typical method for optimum combining of the spread spectrum multipath signal. However, the RAKE receiver, though optimum in single user channel, has inherent limitation in a near-far environment. Recently, quite a few papers have been presented to use multiple antennas for multiuser detection in a wireless communication system, say [2-4]. It is shown in these papers that by exploiting the additional spatial diversity, the capacity, coverage, and quality can be considerably improved. However, they premise on accurate channel parameters' estimate, which is hard to attain in wireless channel anvironment with rich multipath signals. Moreover, since the total number of paths is in general much larger than the number of sensors, the subspace techniques that are widely used in array signal processing problem are inapplicable in the 2D RAKE structure to estimate the channel parameters. A discrete-time model for antenna array CDMA system is formulated in [5] that takes advantage of all possible diversities in a joint fashion. Although this work opened up a new research direction by casting the $2 \mathrm{D}$ RAKE receiver problem into a vector FIR equalizer design and estimation framework, the assumptions that each user's chip delay is known and the spreading codes are binary i.i.d. might be further relaxed for more general consideration.

Toward this purpose, we propose a new and more general spacetime processing structure that fully exploits the potential of the $2 \mathrm{D}$ RAKE receiver without requiring any specific array geometry. This work converts the design of a $2 \mathrm{D}$ RAKE receiver into the design of a vector FIR equalizer or a space-time beamformer. Several highresolution beamforming based multiuser detection strategies are proposed to perform source separation and equalization and the detection algorithm with linear complexity is both computationally and conceptually feasible. It is well known that a robust beamformer that relies on adding pseudo noise can reduce the sensitivity of the performance degradation due to pointing error that arises from the estimation error of the steering vector. However, the anti-near-far problem capability is reduced and the performance degrades since the desired source is regarded as another interferer that the beamformer strives to suppress. Therefore, we propose an adaptive beamforming technique that adaptively modifies the look-direction (estimated steering vector) to the accurate one by maximizing the beamformer's output power while minimizing the projection magnitude on the noise subspace. Simulation results reveal that the proposed adaptive robust beamformer significantly outperforms the beamformer by adding pseudo noise. Instead of estimating the entire channel parameters (including the timings, fading amplitudes, and array response vectors for each path), the proposed structure casts the parameters into a composite form and allows us to benefit from the subspace method to blindly identify the FIR-MIMO channel [6].

\section{Channel model}

In asynchronous DS/CDMA communication system, each user is assigned a unique signature waveform with finite support, $c_{k}(t)=0$, $t \notin[0, T) . T$ is the bit duration. The receiving front end is composed of an array of $M$ antennas (sensors). In mobile communication systems, the time taken for the wavefront to pass through the array is much smaller than the chip interval $T_{c}$ and therefore, the narrowband assumption is valid [2]. Hence, the response of the antenna elements to an arbitrary source is characterized by an array response vector (or steering vector). The steering vector can also be regarded as the spatial signature waveform uniquely specified for each source (path) that emitted from different direction. The multipath environment is modeled by a discrete number of rays each parameterized by a delay, complex amplitude and angle. Therefore, it enables us to model the channel as a multi-input multi-output (MIMO) system. Note that in this framework, different paths for different users are received with different delays, attenuation, and direction of arrival (DOA). The baseband data model of the antenna array output can then be written as $\mathbf{x}(t)=\sum_{k=1}^{K} \sum_{i=1}^{P} \sum_{l=1}^{L_{k}} \alpha_{k l} \sqrt{w_{k}(i)} b_{k}(i) c_{k}\left(t-i T-\tau_{k t}\right) \mathbf{a}_{k l}+\mathbf{v}(t)$

where $\alpha_{k l}$ denotes the complex gain of the $k$ th user's $l$ th path, $\left|\alpha_{k l}\right|<1 . \tau_{k l} \in[0, n)$, is the associated timing offset. $a_{k} \in M \times 1$, denotes the steering vector corresponding to the $k$ th user's $l$ th path, $L_{k}$ denotes the number of paths for $k$ th user and $P T$ is the observation interval. The zero-mean background noise $v(t)$ is assumed to be uncorrelated with the signal and is both temporally and spatially white. Consequently, a space-time receiver designed for extracting the information bits from the array output includes two signal processing aspects:

1. Equalization: to combat ISI arising from the multipath fading.

2. Separation: to discriminate the desired signal against the interferer.

The optimum space-time receiver [4] requires the information of the parameters $\left\{\left\{\tau_{k l}\right\}\left\{a_{k l}\right\}\left\{\sqrt{w_{k}(i)}\right\}\left\{\alpha_{k l}\right\}_{k=1, \ldots, k l=1, \ldots, L_{k}}\right.$ which are 
generally unknown to the receiver. In addition, it is computationally prohibitive which limits the application of the optimum ML receiver especially when $K$ is large.

\section{Linear space-time multiuser receiver}

\section{A. Problem formulation and system model}

It is hard to distinguish each source's transmission by purely space processing (beamforming) for two reasons. First of all, the necessary condition for the sources being identifiable is that the number of sensors is larger than the number of sources [7]. While in the considered multiple access multipath-fading channel, the source number, $\sum_{k=1}^{K} L_{k}$, is in general much larger than the number of sources. Secondly, many high-resolution beamforming techniques require that the second order statistics of the sources being shift-invariant (or widesense stationary (WSS)). However, the DS/CDMA signal, $b_{k}(i) c_{k}(t-i T$ $\left.\tau_{k l}\right)$, is not shift-invariant. Instead, they possess wide-sense "cyclostationarity". By sampling the antenna array's output data chip by chip, then for one bit interval, the discrete-time model can be written as

$$
\begin{aligned}
\mathbf{X}(i) & \left.=\sum_{k=1}^{K} \sum_{l=1}^{L_{k}}+\sqrt{w_{k}(i)} b_{k}(i) \boldsymbol{\alpha}_{k l} \mathbf{a}_{k l} \mathbf{c}_{k l, 0}^{T}\right) \\
& =\sum_{k=1}^{K}\left(\sqrt{w_{k}(i-I)} b_{k}(i-I) \mathbf{S}_{k, 1}+\sqrt{w_{k}(i)} b_{k}(i) \mathbf{S}_{k, 0}\right)+\mathbf{V}(i)
\end{aligned}
$$

where we have assumed that the delay spread is small enough such that only the effects of the most recent two bits are considered. $\tau_{k l}$ is assumed to be positive integer multiples of chip time to simplify the analysis. $\quad \mathbf{c}_{k l, I}=\left[\begin{array}{llllllll}\mathbf{c}_{k}\left(\tau_{k l}\right) & \mathbf{c}_{k}\left(\tau_{k l}+I\right) & \cdots & \mathbf{c}_{k}(N-I) & 0 & 0 & \cdots & 0\end{array}\right]$, $\mathbf{c}_{k, 0}=\left[\begin{array}{llllllll}0 & 0 & \cdots & 0 & \mathbf{c}_{k}(I) & \mathbf{c}_{k}(2) & \cdots & \mathbf{c}_{k}\left(\tau_{k l}-I\right)\end{array}\right]^{\mathrm{a}}$ are the signal vectors associated to the $k$ th user's $l$ th path that $b_{k}(i-l)$ and $b_{k}(i)$ is modulated onto respectively. As depicted in (2), $S_{k, 1}$ is modulated by $b_{k}(i-I)$ and $\mathbf{S}_{k, o}$ is modulated by $b_{k}(i)$. Thus, $\mathbf{S}_{k, l}, \mathbf{S}_{k, 0}$, can be defined as the "space-time signature matrix" of $b_{k}(i-l)$ and $b_{k}(i)$ respectively. Consequently, (2) implies that $\left\{b_{k}(i-l), b_{k}(i)\right\}_{k=1}^{K}$ constitute $2 K$ uncorrelated sources and each source is characterized by a unique space-time signature matrix. It can be regarded as a two-dimensional sensor arrays' processing problem, where the observed data size for each snapshot is $M \times N$. Decomposing the "composite" space-time matrix row- by-row and stacking into a vector with dimension $M N$. Collecting $L$ consecutive measurements, $\{\mathbf{z}(i-L-I), \ldots ., \mathrm{z}(i)\}$, the generalized observation vector, $\mathbf{z}_{L}(i)$, $\mathbf{z}_{L}(i)=\left[\begin{array}{lllll}\mathbf{z}^{H}(i-L-I) & \cdots & \mathbf{z}^{H}(i-I) & \mathbf{z}^{H}(i)\end{array}\right]^{H}$, can be written as

$$
\begin{aligned}
& \mathbf{z}_{L}(i)=\sum_{k=I}^{K}\left[\begin{array}{cccccc|c}
\mathbf{u}_{k}(I) & \mathbf{u}_{k}(0) & 0 & \ldots & \ldots & 0 & s_{k}(i-L) \\
0 & \mathbf{u}_{k}(I) & \mathbf{u}_{k}(0) & 0 & \ldots & \vdots & s_{k}(i-L+D) \\
\vdots & 0 & \ddots & \ddots & \ddots & \vdots \\
\vdots & \ldots & \ddots & \mathbf{u}_{k}(I) & \mathbf{u}_{k}(0) & 0 & s_{k}(i-I) \\
0 & \ldots & \ldots & 0 & \mathbf{u}_{k}(I) & \mathbf{u}_{k}(0) & s_{k}(i)
\end{array}\right]+\mathbf{w}_{L}(i)
\end{aligned}
$$

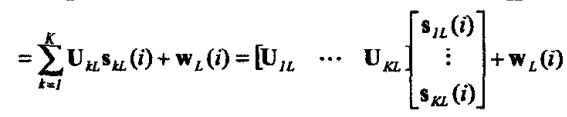

$$
\begin{aligned}
& =\overline{\mathbf{U}}_{L} \tilde{\mathbf{s}}_{L}(i)+\mathrm{w}_{L}(i)
\end{aligned}
$$

The observation vector, $\mathbf{z}_{L}(i)$, can be obtained by first sampling each sensor's output chip-by-chip, buffering each chip matched filter's output data sequence into blocks of length $N$, and then stacking all sensors' output data block into observation vectors of length $M N L$ as depicted in Fig. 1. It should be noted that (3) leads to a beamforming framework. $\mathbf{z}_{L}(i)$ denotes the data extracted from array of sensors of size $M N L$ at the $i$ th snapshot. Each column of $\tilde{\mathbf{U}}_{L}$ stands for the steering vector that corresponds to $K(L+1)$ uncorrelated sources, $\left\{b_{k}(i-L), \ldots b_{k}(i-1), b_{k}(i)\right\}_{k=1}^{k}$. Since the steering vectors arise from a composite form of the spatial and temporal parameters, hence the beamformer designed based on the proposed structure can be defined as the "space-time beamformer". Different from the 2-D RAKE receiver, the spatial diversity, $M$, temporal diversity, $N$, and $L$ are jointly exploited in this framework. Toward this end, we can apply several beamforming techniques to extract the desired signal and reject the interference and noise.

\section{B. Space-time beamforming techniques B.1 Minimum variance distortionless response (MVDR) beamformer}

If we collect the measurements over two bits interval $(L=2)$, the measurement equation can be obtained by substituting $L=2$ into (3). The goal is to optimize the beamformer's response such that the output contains minimal contributions from noise and signals arriving from "directions" other than the desired signal's "direction". Note that its unique signal (steering) vector that presented in a composite form of the spatial and temporal parameters characterizes the "direction" of each source. Assuming that the desired user's steering vector can be perfectly estimated, i.e., $\mathbf{u}_{k}(0), \mathbf{u}_{k}(l)$ are known, then the criterion of the MVDR beamformer leads to the constrained optimization problem: $\left\{\begin{array}{l}\arg \underset{\mathbf{t}_{\text {(MANDR })}}{\min } E\left\{\left.\mathbf{t}_{k(M V D R)}^{H} \mathbf{z}_{2}\right|^{2}\right\}=\arg \underset{\mathbf{t}_{\text {kMVDR }}}{\min }\left\{\mathbf{t}_{k(M V D R)}^{H} \mathbf{R}_{2} \mathbf{t}_{k(M V D R)}\right\} \\ \text { subject to } \quad \mathbf{t}_{k(M V D R)}^{H} \mathbf{u}_{k,-1}=1\end{array}\right.$

where $\mathbf{R}_{2}=E\left\{\mathbf{z}_{2}(i) \mathbf{z}_{2}^{H}(i)\right\}$. The solution of (4) can be obtained from [8]

$\mathbf{t}_{k(M V D)}=\lambda_{k(M V D R)} \mathbf{R}_{2}^{-1} \mathbf{u}_{k,-1} \quad ; k=1, \ldots \ldots, K$

where $\lambda_{k(M V D R)}$ denotes the output power of the MVDR beamformer.

$\lambda_{k(M V D R)}=\mathbf{t}_{k(M V D R)}^{H} \mathbf{R}_{2} \mathbf{t}_{k(M V D R)}=\frac{1}{\mathbf{u}_{k,-l}^{H} \mathbf{R}_{2}^{-1} \mathbf{u}_{k,-1}} ; k=1 \ldots ., K$

If user $l$ is the desired user that transmits BPSK information bits. Then, information bits can be estimated by passing the beamformer's output to a threshold device. The bit error probability of user l's ( $i$ l)th bit, $\hat{b}_{1}(i-1)$, can be written as

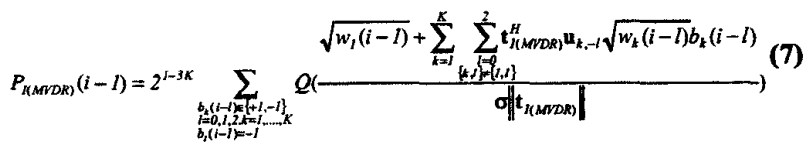

\section{B.2 Multiply constrained minimum variance (MCMV) beamformer}

An alternative beamforming technique can be modified from (4) by adding auxiliary constraints to suppress interfering signals in a hard manner. It is defined as multiply constrained minimum variance (MCMV) beamformer [8] since several linear constraints are included in the criterion to pass the desired signal and simultaneously place nulls in the directions of some interfering sources. 


$$
\left\{\begin{array}{c}
\underset{\left.\mathbf{t}_{\mathbf{k}(M C W V}\right)}{\arg \min } E\left\{\left.\mathbf{t}_{k(M C M V)}^{H} \mathbf{z}_{2}\right|^{2}\right\}=\arg \min _{\mathbf{t}_{\mathbf{k}(M C W V}}\left\{\mathbf{t}_{k(M C M V)}^{H} \mathbf{R}_{2} \mathbf{t}_{k(M C M V)}\right\} \\
\text { subject to } \quad \begin{array}{l}
\mathbf{t}_{k(M C M V)}^{H} \mathbf{u}_{k,-l}=l \\
\mathbf{t}_{k(M C M V)}^{H} \mathbf{u}_{j,-l}=0
\end{array} \quad ; j, k=1, \ldots, K \quad l=0,1,2
\end{array}\right.
$$

where the first constraint forces the desired signal (with steering vector $\mathbf{u}_{k,-l}$ ) to be distortionlessly passed, and the second constraint places nulls to suppress the interfering signals. In particular, to perform the MCMV beamforming, one should further know the steering vectors of the interferers that are needed to be nulled out. Major disadvantage of the MCMV beamformer is the "noise enhancement" problem, which resulted from the case that the beamformer places nulls to the low SNR interferers. Intuitively, the MVDR beamformer places deep nulls to those interferers with high SNR while places shallow nulls to those interferers with low SNR to avoid "noise enhancement". Only the information of the desired user's steering vector should be $a$ priori known, the MVDR beamformer can automatically suppress interference and background noise. It can be derived that the weight vector of the MVDR beamformer reduces to the MCMV beamformer in the absence of background noise. As background noise is considered, the MVDR beamforming based detector outperforms the MCMV beamforming based detector since it avoids the noise enhancement effect. Note that both the MVDR detector and the MCMV detectors are with linear complexity and the computation load is comprehensively reduced compared to the 2D RAKE receiver.

\section{Robust space-time multiuser receiver}

This section describes the effects of mismatch or pointing error (estimation error of the steering vector) on system performance. Moreover, we will design a robust MVDR detector that comprehensively improves the performance degradation resulted from pointing error.

\section{A. Effects of pointing error}

Intuitively, the steering vector can be regarded as the DOA for each source. The MVDR detector distortionlessly passes signal in the "look direction", $\mathbf{u}_{k,-l}$, while automatically suppress interference and background noise other than the look direction. Any signal that is not exactly matched to the look-direction is treated as an unwanted interfering signal by the MVDR beamformer and therefore tends to be suppressed. The degree of suppression depends on the desired user's SNR. Consequently, the beamformer's output power drops as a result of pointing error and the suppression of the desired signal leads to severe performance degradation. It is shown in [9] that the MVDR beamformer is highly sensitive to pointing error especially for larger array size (the effective number of sensors).

\section{B. Robust MVDR beamforming by adding pseudo noise}

Intuitively, if we intend to reduce the desired source's effective SNR (seen by the beamformer), it may get less suppressed. We intend to lower down input SNR by adding pseudo noise into $\mathbf{R}_{2}$

$$
\tilde{\mathbf{R}}_{2}=\mathbf{R}_{2}+\sigma_{P}^{2} \mathbf{I}_{2 M N}=\tilde{\mathbf{U}}_{2} \mathbf{W} \tilde{\mathbf{U}}_{2}^{H}+\left(\sigma_{P}^{2}+\sigma^{2}\right) \mathbf{I}_{2 M N}
$$

where $\sigma_{P}^{2}$ is the pseudo noise power (variance). An adaptive multiuser detection structure to mitigate the effect of mismatch is presented in [10], which relies on tuning the value of the pseudo noise variance.
However, the robustness is attained at the expense of anti-near-far problem capability [10]. It can be realized since the interferers' SNRs have also been reduced by the pseudo noise, the MVDR beamformer tends to place shallower nulls to eliminate the interference, which apparently degrades the anti-near far problem capability. Moreover, the performance improvement by adding pseudo noise is expected to be very limited. This is due to the fact that the desired source is inherently treated as another interferer that the MVDR beamformer strives to suppress.

\section{Adaptive robust beamforming technique}

Exploiting the subspace method and the variation of the MVDR beamformer's output power, we propose an algorithm in which the pointing error can be adaptively minimized. Therefore, the lookdirection, can be adaptively tuned to the actual one $u_{k,+1}$.

\section{C.1 Preliminary}

\section{C.1.1 Subspace method}

Performing eigendecomposition (EVD) on $\mathbf{R}_{2}$ and decomposing the eigenvectors into signal subspace, $\mathbf{E}_{s}$, and noise subspace $\mathbf{E}_{n}$, we can obtain from [11] that the signal vectors $\left\{\mathbf{u}_{k,-1}\right\}_{k=1}^{K}$ should be orthogonal to $\mathbf{E}_{n}$. Therefore, the first performance criterion to be minimized is the projection magnitude of $\hat{\mathbf{u}}_{k,-1}$ onto $\mathbf{E}_{n}$.

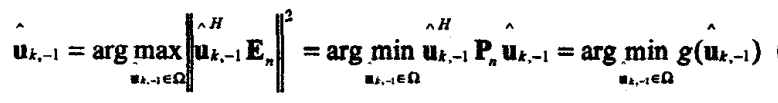

where $\Omega$ is the constraint set that places on $\hat{\mathbf{w}}_{k,-l}$ of the searching algorithm to avoid trivial solution. $\mathbf{P}_{n}=\mathbf{E}_{n} \mathbf{E}_{n}^{H}$ is the projection matrix that projects onto the noise subspace. It is reasonable to set the constraint that the look direction, $\hat{\mathbf{u}}_{k,-1}$, is in the vicinity of $\mathbf{u}_{k-1}$, or equivalently, it is closer to the desired source's actual direction than the directions associated to other interferers.

\section{C.1.2 MVDR beamformer's output power}

If $\hat{\mathbf{u}}_{k,-I}$ is perfectly matched to $\mathbf{u}_{k,-l}$, the desired signal is distortionlessly passed such that the output power increases as the desired signal's SNR increases. Whereas, as "pointing error" occurs, the output power remains insensitive to the variation of the desired signal's SNR. This is due to the fact that the stronger the desired user is, it gets more suppressed. The MVDR beamformer's output power with look-direction $\hat{\mathbf{u}}_{k,-1}$ should be less than the one with look direction exactly matched to $\mathbf{u}_{k,-1}$. It can be realized since the MVDR beamformer suppresses the desired signal while preserving the power coming from $\hat{\mathbf{u}}_{k_{1}-1}$, which contains background noise only. Motivated by the output power variation, we propose to adapt $\hat{\mathbf{u}}_{k,-l}$ toward the direction of maximizing the MVDR beamformer's output power as the second performance criterion. We can obtain from (6)

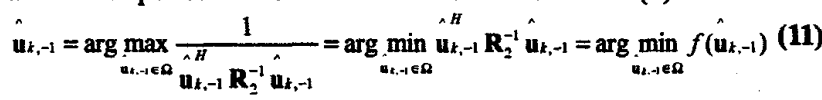

\section{C.2 Adaptive algorithm}

To develop the adaptive algorithm, we can use $\hat{\mathbf{u}}_{k,-1}$ as the initial guess, $\hat{\mathbf{u}}_{k,-1}(0)$. Then the algorithm adaptively tunes $\hat{\mathbf{u}}_{k,-1}(0)$ 
toward the direction of maximizing the beamformer's output power while minimizing the projection magnitude in each step of $\hat{\mathbf{u}}_{k,-1}(m)$, $m=0,1, \ldots$ onto the noise subspace. In particular, $\hat{\mathbf{u}}_{k,-1}(m)$ is within the constraint set that it is in the vicinity of $\mathbf{u}_{k,-1}$. Combining (11) and (10) and applying the gradient search method, the algorithm can be realized in the following steps: For $m=0,1,2, \ldots$

step I:

$$
\begin{aligned}
\hat{\mathbf{u}}_{k,-I}(m+I) & =\hat{\mathbf{u}}_{k,-I}(m)-\left.\beta \nabla_{\hat{\mathbf{u}}_{k,-1}}\left(f\left(\hat{\mathbf{u}}_{k,-1}\right)\right)\right|_{\mathbf{u}_{k,-l}=\mathbf{u}_{k,-l}(m)} \\
& =\hat{\mathbf{u}}_{k,-l}(m)-\beta \mathbf{R}_{2}^{-1} \hat{\mathbf{u}}_{k,-I}(m)
\end{aligned}
$$

step 2:

$$
\begin{aligned}
\hat{\mathbf{u}_{k,-l}(m+2)} & =\hat{\mathbf{u}}_{k,-l}(m+I)-\left.\delta \nabla_{\hat{\mathbf{u}}_{k,-l}}\left(g\left(\hat{\mathbf{u}}_{k_{,-1}}\right)\right)\right|_{\mathbf{u}_{k,-l}=\mathbf{u}_{k,-l}(m+l)} \\
& =\hat{\mathbf{u}}_{k,-I}(m+I)-\delta \mathbf{P}_{n} \hat{\mathbf{u}}_{k,-l}(m+I)
\end{aligned}
$$

step 3: If $\left\|\hat{\mathbf{u}}_{k,-I}(m+2)-\hat{\mathbf{u}}_{k,-l}(0)\right\|^{2}>\varepsilon$, stop, otherwise, return to step 1 .

where $\beta, \delta$, are the step sizes to control the speed of the algorithm. $\varepsilon$ is chosen to stop the adaptation such that $\hat{\mathbf{u}}_{k,-1}(m+2)$ is constrained to be within the vicinity of $\mathbf{u}_{k,-1}$. Note that step 1 forces $\hat{u}_{k,-1}(m)$ to $\hat{\mathbf{u}}_{k,-l}(m+I)$ in the direction of maximizing the beamformer's output power, while step 2 forces $\hat{\mathbf{u}}_{k,-I}(m+I)$ to $\hat{\mathbf{u}}_{k,-l}(m+2)$ in order to minimize the projection magnitude on the noise subspace. Step 3 controls the iteration number of the adaptation algorithm.

\section{Performance evaluation}

It is assumed that two users each with three distinct paths are received by a uniform linear array. Gold code with $N=7$ are used. The delays and fading parameters for each user and each path are: $\tau_{11}=2$, $\tau_{12}=3, \quad \tau_{13}=5, \quad \tau_{21}=3, \quad \tau_{22}=4, \quad \tau_{23}=6 \quad T_{c}, \quad \alpha_{11}=\sqrt{0.6}, \quad \alpha_{12}=\sqrt{0.3}$, $\alpha_{13}=\sqrt{0.1}, \alpha_{21}=\sqrt{0.7}, \alpha_{22}=\sqrt{0.2}, \alpha_{23}=\sqrt{0.1}$. The arriving angles for each path are $\theta_{11}=60^{\circ}, \theta_{12}=90^{\circ}, \theta_{13}=108^{\circ}$, $\theta_{2 I}=30^{\circ} \quad \theta_{22}=45^{\circ}, \theta_{23}=120^{\circ}$ respectively. Assuming that the composite steering vectors have been perfectly estimated, we compare the bit error probability of the conventional, MVDR and MCMV detectors with respect to the interferer to desired user's power ratio in Fig. 2. As shown in Fig. 2, the MVDR detector outperforms the MCMV detector especially when the MAI effect is not severe. This is due to the "noise enhancement" effect. Also from Fig. 2, the error probability of the MVDR detector approaches to the MCMV detector as the interferer's power increases. This verifies the fact that the MVDR and MCMV beamformer are equivalent in the absence of noise. Fig. 3 characterizes the performance improvement by increasing the size of the antenna array (spatial diversity). To evaluate the effect of pointing error on system performance, it is assumed that the estimated steering vector, $\hat{\mathbf{u}}_{k,-l}$, deviates from $\mathbf{u}_{k,-l}$ by a zero-mean Gaussian random vector, $\mathbf{d}_{k}$. $\hat{\mathbf{u}}_{k,-l}=\mathbf{u}_{k,-l}+\mathbf{d}_{k}$. Effect of pointing error on system performance is presented in Fig. 4 (a) $\sim(c)$. One can observe from Fig. 4(b) and (c) that the performance of the MVDR and
MCMV detectors are severely degraded as pointing error occurs. It can be realized since as pointing error occurs, both the MVDR and MCMV detectors pass the signal coming from look direction, $\hat{\mathbf{u}}_{k,-1}$, whereas, it is full of noise. We further compare the performance of the robust MVDR detector (by adding pseudo noise) and the proposed adaptive robust beamforming structure in Fig. 5(a). It is shown that the BER of the ideal MVDR detector (without pointing error) decreases as the desired signal's SNR (SNR1) increases. Whereas, poor performance can be observed in the case with pointing error no matter how large SNR1 is. The robust MVDR detector though slightly better than the case with pointing error, cannot improve system performance in accordance with SNRI. This is due to the fact that adding pseudo noise only reduce the effective SNR while the desired source is inherently being treated as an interferer to be suppressed. As we adaptively modify $\hat{\mathbf{u}}_{k,-1}$ toward $\mathbf{u}_{k_{1}-l}$, the performance has been comprehensively improved. Moreover, the performance improves in accordance with SNR1 and approaches the ideal case. Fig. 5(b) presents the variation of MVDR beamformer's output power with respect to SNR1. As expected, the output power increases as SNR1 increases for the ideal case and the adaptive robust beamformer. Whereas, the case with pointing error and the method by adding pseudo noise are both insensitive to the variation of SNR1. From Fig. 5 , we have verified that the proposed adaptive algorithm has effectively improved system performance and approaches the ideal case. In particular, the adaptation algorithm is near-far resistant since both the noise subspace and the beamformer's output power is not affected by the interferers' power.

\section{Conclusion}

In this work, we have proposed a new space-time processing structure that simultaneously exploits both the spatial and temporal diversities for the antenna CDMA system. Several high-resolution beamforming techniques including the MVDR and MCMV beamformers can be applied to perform equalization and source separation with reliable performance and linear complexity. It is shown that the proposed adaptive robust beamformer greatly outperforms for the case by adding pseudo noise and approaches the performance of the ideal MVDR beamformer. Furthermore, the proposed space-time processing structure is appropriate to apply the high-resolution subspace method for channel parameters' estimation. Therefore, we can conclude from the above discussion that the proposed simple and reliable space-time processing strategy is very attractive in practical wireless CDMA communications.

\section{References}

[1] R. Price and Jr. P.E. Green, "A communication technique for multipath channels" Proc. IRE, 46:555-570, March 1958.

[2] H. Khalaj, A. Paulraj, and T. Kailath, "2-D RAKE receiver for CDMA cellular system", IEEE Globecom'94, pp. 400-404, San Francisco, USA, 1994.

[3] A.J. Paulraj and C.B. Papadias, "Space-time processing for wireless communications" IEEE Signal Processing Magazine, pp. 4983, Nov. 1997.

[4] A.F. Naguib, "Space-time receivers for CDMA multipath signals" IEEE Signal Processing Letts. pp.304-308, Nov. 1997.

[5] H. Liu, and M.D. Zoltowski, "Blind equalization in antenna array 
CDMA systems" IEEE Trans. Signal Processing, Vol. 45, No. 1, pp. 161-172, Jan. 1997.

[6] S. E. Bensley and B. Aazhang, "Subspace-based channel estimation for code division multiple access communication system" IEEE Trans. Commun., Vol. 44, No. 8, pp. 1009-1020, Aug. 1996.

[7]P. Stoica and A. Nehorai, "MUSIC, maximum likelihood and Cramer-Rao bound" IEEE Trans. Acoust. Speech Signal Processing, ASSP-37: 720-741, May 1989.

[8] B. D. Van Veen and K. M. Buckley, "Beamforming: A versatile approach to spatial filtering" IEEE ASSP Magazine, pp.4-24, April, 1988.

[9] A.K. Steele, "Comparison of directional and derivative constraints for beamformer subject to multiple linear constraints" IEE Proceeding-F, Vol.130, No. 1, pp. 41-45, Feb. 1983.

[10] M.L. Honig, U. Madhow, and S. Verdu, "Blind adaptive multiuser detection" IEEE Trans. Inform. Theory, Vol. 41, pp. 944966, July 1995.

[11] W.C. Wu and K.C. Chen, "Root-MUSIC based joint identification and timing estimation of asynchronous CDMA system over Rayleigh fading channel" IEICE Transaction on Fundamentals of Electronics, Communications and Computer Sciences, vol. E81-A, no.8, pp. 1550-1559, Aug. 1998.

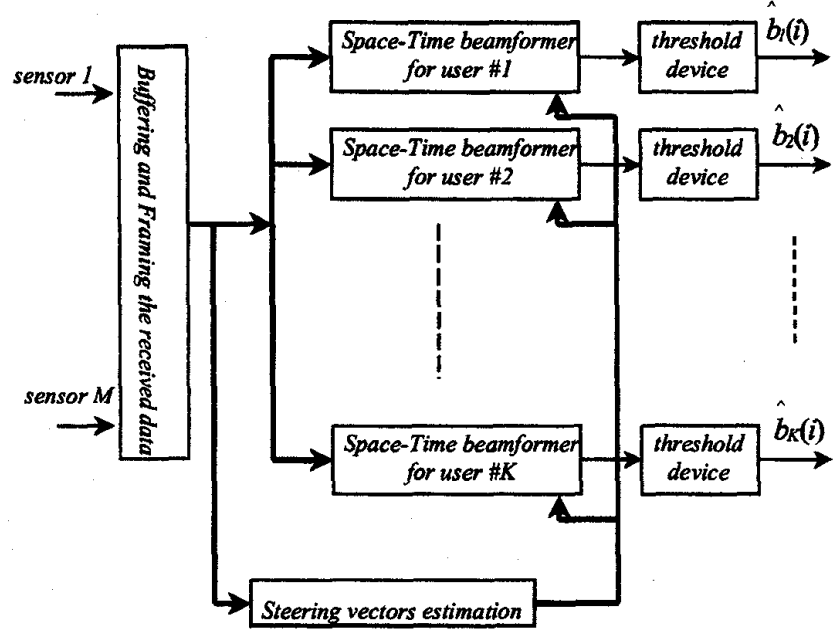

Fig. 1: Structure of the proposed linear Space-Time multiuser receiver

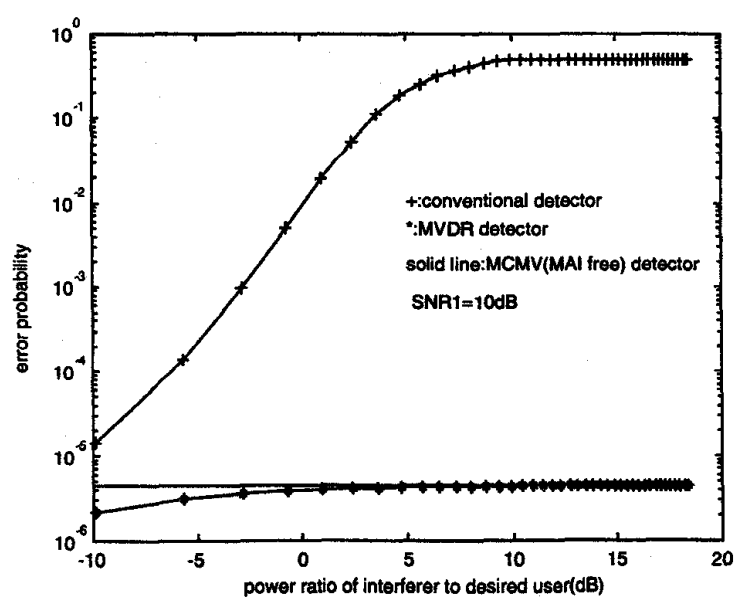

Fig. 2: Performance comparison of the conventional, MVDR, and MCMV detectors in terms of BER

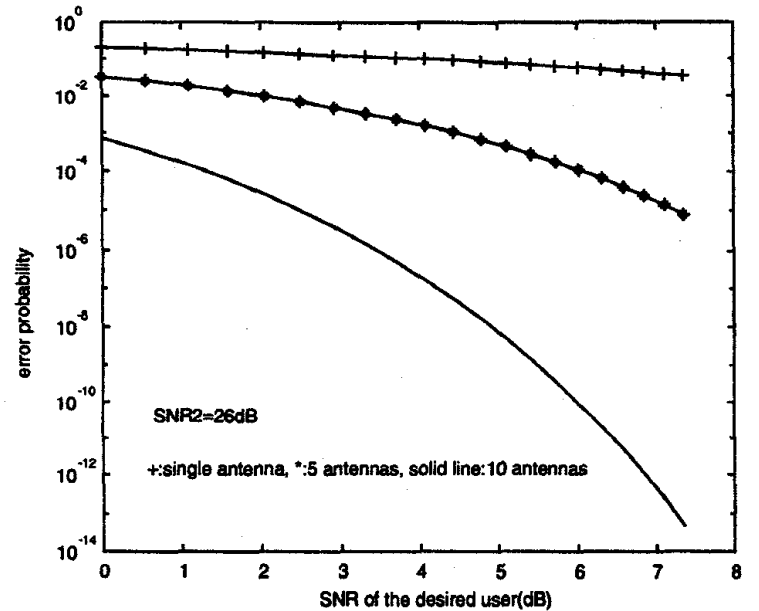

Fig. 3: Pefformance comparison among different size of antenna arrays
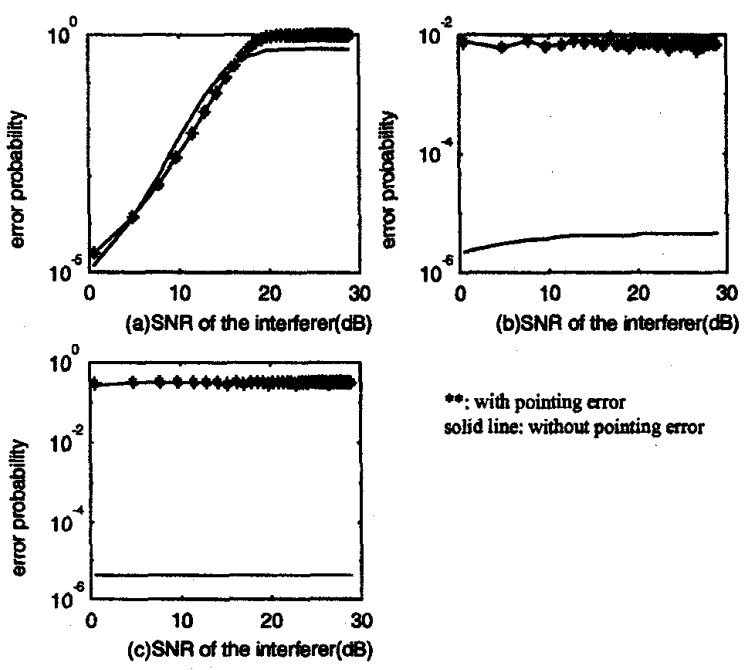

*: with pointing error solid line: without pointing error

Fig. 4: Effect of pointing error on system performance. (a) Conventional detector (b) MVDR detector (c) MCMV detector
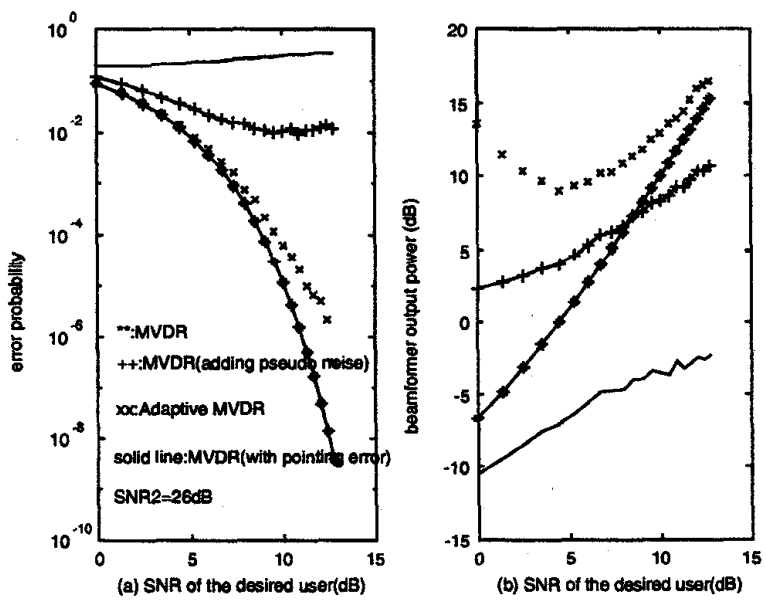

Fig. 5: (a) Peformance conpericon of the MVDR detector for the ideal case, with pointimg arox robust MVDR beamformer (by adding pseudo noise), and adaptive MVDR beamformer robust MVDR bearnformer (by adding pseudo noise), and adaptive MVDR beamforme b) Comparison of the MVDR beamformer's output power for the ideal case, with pointing 